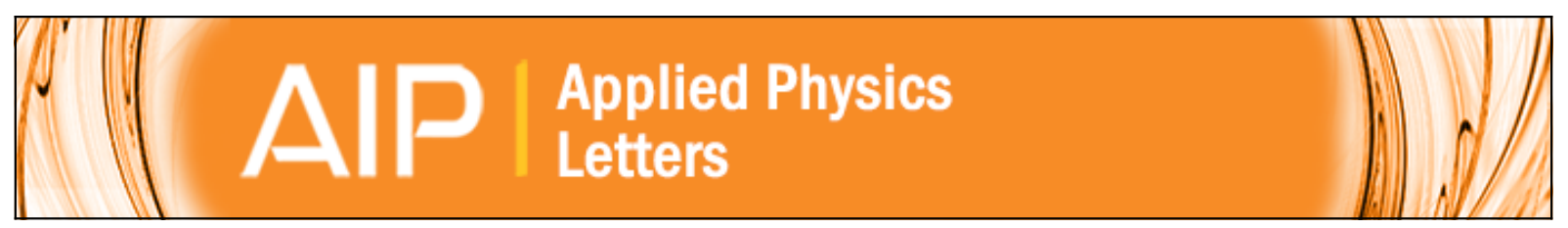

\title{
Admittance spectroscopy of Cu2ZnSnS4 based thin film solar cells
}

P. A. Fernandes, A. F. Sartori, P. M. P. Salomé, J. Malaquias, A. F. da Cunha, M. P. F. Graça, and J. C.

González

Citation: Applied Physics Letters 100, 233504 (2012); doi: 10.1063/1.4726042

View online: http://dx.doi.org/10.1063/1.4726042

View Table of Contents: http://scitation.aip.org/content/aip/journal/apl/100/23?ver=pdfcov

Published by the AIP Publishing

\section{Articles you may be interested in}

Rapid thermal annealed Molybdenum back contact for Cu2ZnSnS4 thin film solar cells

Appl. Phys. Lett. 106, 131110 (2015); 10.1063/1.4916994

Comparison of fluctuating potentials and donor-acceptor pair transitions in a Cu-poor Cu2ZnSnS4 based solar cell

Appl. Phys. Lett. 105, 163901 (2014); 10.1063/1.4899057

Relationship between Cu2ZnSnS4 quasi donor-acceptor pair density and solar cell efficiency

Appl. Phys. Lett. 103, 193903 (2013); 10.1063/1.4829920

Toward a high Cu2ZnSnS4 solar cell efficiency processed by spray pyrolysis method

J. Renewable Sustainable Energy 5, 053137 (2013); 10.1063/1.4825253

Impact of KCN etching on the chemical and electronic surface structure of Cu2ZnSnS4 thin-film solar cell absorbers

Appl. Phys. Lett. 99, 152111 (2011); 10.1063/1.3650717

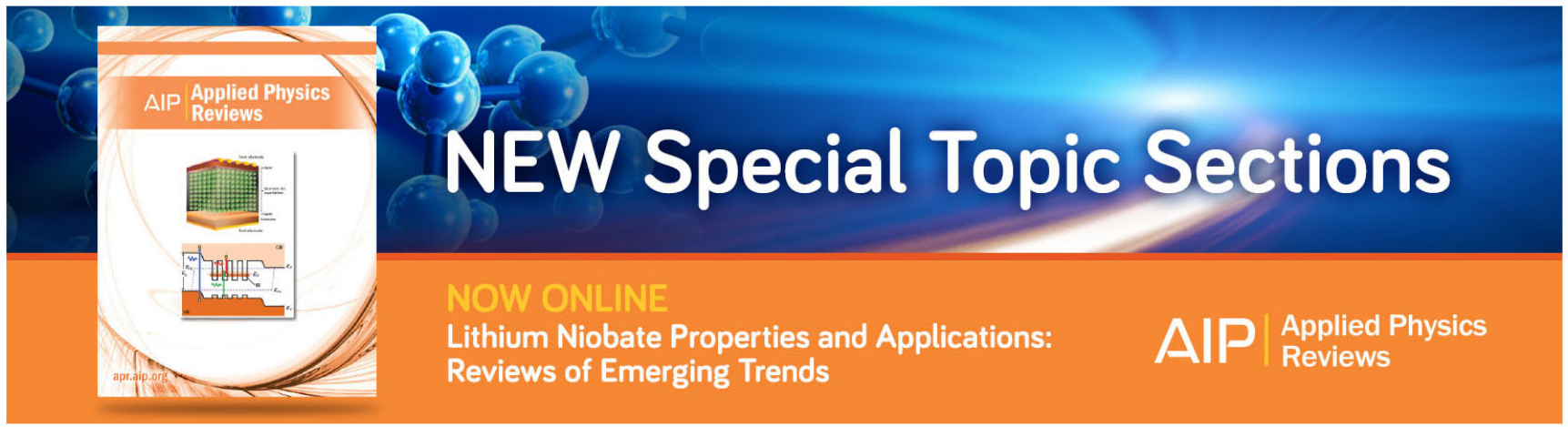




\title{
Admittance spectroscopy of $\mathrm{Cu}_{2} \mathrm{ZnSnS}_{4}$ based thin film solar cells
}

\author{
P. A. Fernandes, ${ }^{1,2, a)}$ A. F. Sartori, ${ }^{1}$ P. M. P. Salomé, ${ }^{1}$ J. Malaquias, ${ }^{1}$ A. F. da Cunha, ${ }^{1}$ \\ M. P. F. Graça, ${ }^{1}$ and J. C. González ${ }^{3}$ \\ ${ }^{1}$ I3N and Departamento de Física, Universidade de Aveiro, Campus Universitário de Santiago, \\ 3810-193 Aveiro, Portugal \\ ${ }^{2}$ Departamento de Física, Instituto Superior de Engenharia do Porto, Instituto Politécnico do Porto, \\ Rua Dr. António Bernardino de Almeida, 431, 4200-072 Porto, Portugal \\ ${ }^{3}$ Departamento de Física, Universidade Federal de Minas Gerais, 30123-970 Belo Horizonte, \\ Minas Gerais, Brazil
}

(Received 16 February 2012; accepted 21 May 2012; published online 6 June 2012)

\begin{abstract}
In this report, we propose an $\mathrm{AC}$ response equivalent circuit model to describe the admittance measurements of $\mathrm{Cu}_{2} \mathrm{ZnSnS}_{4}$ thin film solar cell grown by sulphurization of stacked metallic precursors. This circuit describes the contact resistances, the back contact, and the heterojunction with two trap levels. The study of the back contact resistance allowed the estimation of a back contact barrier of $246 \mathrm{meV}$. The analysis of the trap series with varying temperature revealed defect activation energies of $45 \mathrm{meV}$ and $113 \mathrm{meV}$. The solar cell's electrical parameters were obtained from the J-V curve: conversion efficiency, 1.21\%; fill factor, 50\%; open circuit voltage, $360 \mathrm{mV}$; and short circuit current density, $6.8 \mathrm{~mA} / \mathrm{cm}^{2}$. (C) 2012 American Institute of Physics. [http://dx.doi.org/10.1063/1.4726042]
\end{abstract}

Despite the success reached by CdTe and $\mathrm{CuIn}_{1-x} \mathrm{Ga}_{x} \mathrm{Se}_{2}$ (CIGS) based thin film solar cells (TFSC), toxicity and scarcity problems may represent a limiting factor for large scale production in the future. This is one of the reasons why an increasing number of research groups are putting effort in developing and studying alternative absorber layers such as $\mathrm{Cu}_{2} \mathrm{ZnSnS}_{4}$ (CZTS). So far, for pure sulfur CZTS the highest published efficiency is $8.4 \%$ using co-evaporation techniques. ${ }^{1}$ Using an absorber layer based on a chalcogenide mixture of $\mathrm{S}$ and $\mathrm{Se}$, Barkhouse et al. published the highest efficiency reached by this family of compounds, $10.1 \%$, using non-vacuum growth methods. ${ }^{2}$ In order to have a better understanding of the factors that are limiting the achievement of higher efficiencies, the device's electrical characterization must be carried out. The study of the open circuit voltage vs. temperature, the external quantum efficiency for different bias voltages and time-resolved photoluminescence allows a clearer understanding of these limiting factors. ${ }^{1,3,4}$ In this work, we use admittance spectroscopy to perform the electrical characterization of the solar cell. This technique was already used on CZTS based devices grown by co-evaporation methods and also on CdTe and CIGS solar cells. ${ }^{2,5,6}$ With this technique, an $\mathrm{AC}$ response equivalent circuit was proposed and from the resulting model several parameters were extracted, allowing the characterization of the CZTS/CdS heterojunction and the $\mathrm{Mo} / \mathrm{MoS}_{2} / \mathrm{CZTS}$ back contact interfaces.

The absorber layer and solar cell were grown according to a method described elsewhere. ${ }^{7}$ The composition was analyzed using energy dispersive spectroscopy measurements of the top surface of the absorber layer. Metal composition ratios, $[\mathrm{Cu}] /[\mathrm{Zn}]+[\mathrm{Sn}]$ and $[\mathrm{Zn}] /[\mathrm{Sn}]$, in accordance to the value that provides an optimum electrical performance as shown by Katagiri et al. in an empirical study, ${ }^{8} 0.8$ and 1.2, respectively. For phase detection, two techniques were

${ }^{a)}$ Electronic mail: pafernandes@ua.pt. employed, x-ray diffraction and Raman scattering. ${ }^{9}$ These measurements have shown that the dominating phase is CZTS, but Raman peak signatures of $\beta$-ZnS and cubic$\mathrm{Cu}_{2} \mathrm{SnS}_{3}$ were also found. ${ }^{10}$ Optical transmittance and reflectance measurements were performed on samples without the Mo layer, allowing the estimation of the CZTS band gap to be $1.50 \mathrm{eV}$. The parameters obtained from the $\mathrm{J}-\mathrm{V}$ curve are summarized in Table I. Interface and bulk recombination centers, formation of secondary phases, and poor heterointerface quality are, probably, the sources of this low performance.

The admittance measurements were performed in a homemade cryostat with temperatures ranging $80 \mathrm{~K}$ to $360 \mathrm{~K}$ using an Agilent 4294A precision impedance analyzer and an Oxford ITC4 temperature controller. All data were acquired in dark conditions with no applied bias. The amplitude of the AC signal was set to be constant and equal to $25 \mathrm{mV}$ and the analyzed frequency range was $40 \mathrm{~Hz}$ to $1 \mathrm{MHz}$.

Fig. 1 shows the collected imaginary part of the admittance, $\operatorname{Im}[Y(\omega)]$, vs. the real part of the admittance, $\operatorname{Re}[Y(\omega)]$, for temperatures of $293 \mathrm{~K}, 320 \mathrm{~K}$, and $360 \mathrm{~K}$. The curves for lower temperatures followed the same trend. This figure shows semicircles increasing with decreasing temperature. This is a consequence of the thermally activated behavior of the conductivity. 5

Fig. 2(a) shows the three equivalent circuits tested to adjust the admittance data, namely M1, M2, and M3. The fitting of the model to the measured data was done with ZSimpWin 3.22d. ${ }^{11}$ The simplest model, M1, corresponds to

TABLE I. Electrical parameters for the studied solar cell.

\begin{tabular}{lcccccc}
\hline \hline $\begin{array}{c}\text { Eff. } \\
(\%)\end{array}$ & $\begin{array}{c}\mathrm{FF} \\
(\%)\end{array}$ & $\begin{array}{c}J_{s c} \\
\left(\mathrm{~mA} / \mathrm{cm}^{2}\right)\end{array}$ & $\begin{array}{c}V_{o c} \\
(\mathrm{mV})\end{array}$ & $\begin{array}{c}R_{s} \\
\left(\Omega \mathrm{cm}^{2}\right)\end{array}$ & $\begin{array}{c}R_{p} \\
\left(\Omega \mathrm{cm}^{2}\right)\end{array}$ & $\begin{array}{c}\mathrm{A} \\
(\text { dark/light })\end{array}$ \\
\hline 1.2 & 50 & 6.8 & 360 & 0.85 & 360 & $1.9 / 2.1$ \\
\hline \hline
\end{tabular}




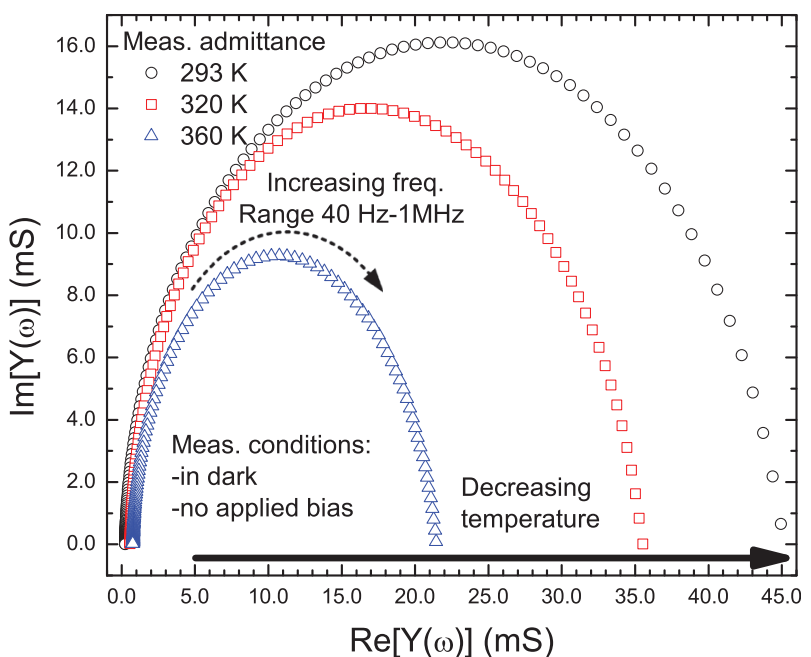

FIG. 1. (a) Complex admittance plane of the studied solar cell measured at temperature of $293 \mathrm{~K}, 320 \mathrm{~K}$, and $360 \mathrm{~K}$, in dark conditions with no applied bias voltage.

a device characterized by a series resistance, $R_{s}$, and a parallel capacitance and resistance, $C_{j}$ and $R_{j}$, respectively. The first element models all the contact and material resistances, such as Mo contact, $\mathrm{ZnO}: \mathrm{Al}$ front window and $\mathrm{Ni}: \mathrm{Al}$ contact grid and the loop describes the CZTS/CdS heterojunction AC response. An extra loop is added for the circuit M2, formed by $R_{b}$ and $C_{b}$ which models the behaviour of a nonohmic $\mathrm{Mo}-\mathrm{MoS}_{2} / \mathrm{CZTS}$ electrical back contact. In the last model, M3, two capacitor-resistor $\left(C_{1}-R_{1}, C_{2}-R_{2}\right)$ pairs have been added to the CZTS/CdS network to account for recombination centers in the CZTS layer. ${ }^{5}$ Additional C-R pairs in the CdS/CZTS heterojunction section did not show visible improvements in the fittings results. ${ }^{12}$

Fig. 2(b) shows the measured admittance for a temperature of $293 \mathrm{~K}$ and the fitting results for the three models. The fitting deviations for the admittance module, $|Y|$, versus frequency are shown in Fig. 2(c). These results show that model M3 is the most suited one. For a low frequency regime, $f<500 \mathrm{~Hz}$, all models present satisfactory fitting results with deviations smaller than 5\%. In this regime, the AC response of the device is dominated by the capacitance of the junction, $C_{j} .{ }^{13,14}$ To improve the fittings for a higher frequency regime, the back contact loop must be added, as shown in model M2 and M3. Note that the maximum fitting deviation decreases from $\sim 25 \%$ (M1) to $\sim 8 \%$ (M2). In this regime, the capacitance behavior of the solar cell is given by the relation $C \sim\left(1 / C_{j}+1 / C_{b}\right)^{-1} \cdot{ }^{13,14}$ The inclusion of the trap states reduces the error to below 2\% (M3). Similar results were obtained for the other temperatures.

The extracted resistances values, $R_{b}, R_{j}, R_{1}$, and $R_{2}$, are presented in Fig. 3(a). All these parameters show an exponential increase with decreasing temperature, as shown in the logarithmic plot in Fig. 3(a). $R_{j}$ seems to be well fitted using the relation $1 / R_{j}=G_{j}=G_{0} \times \exp \left(T / T_{0}\right)$, where $G_{j}$ is the junction conductance, $G_{0}$ and $T_{0}$ are constants. This behavior is known as temperature assisted tunneling and is
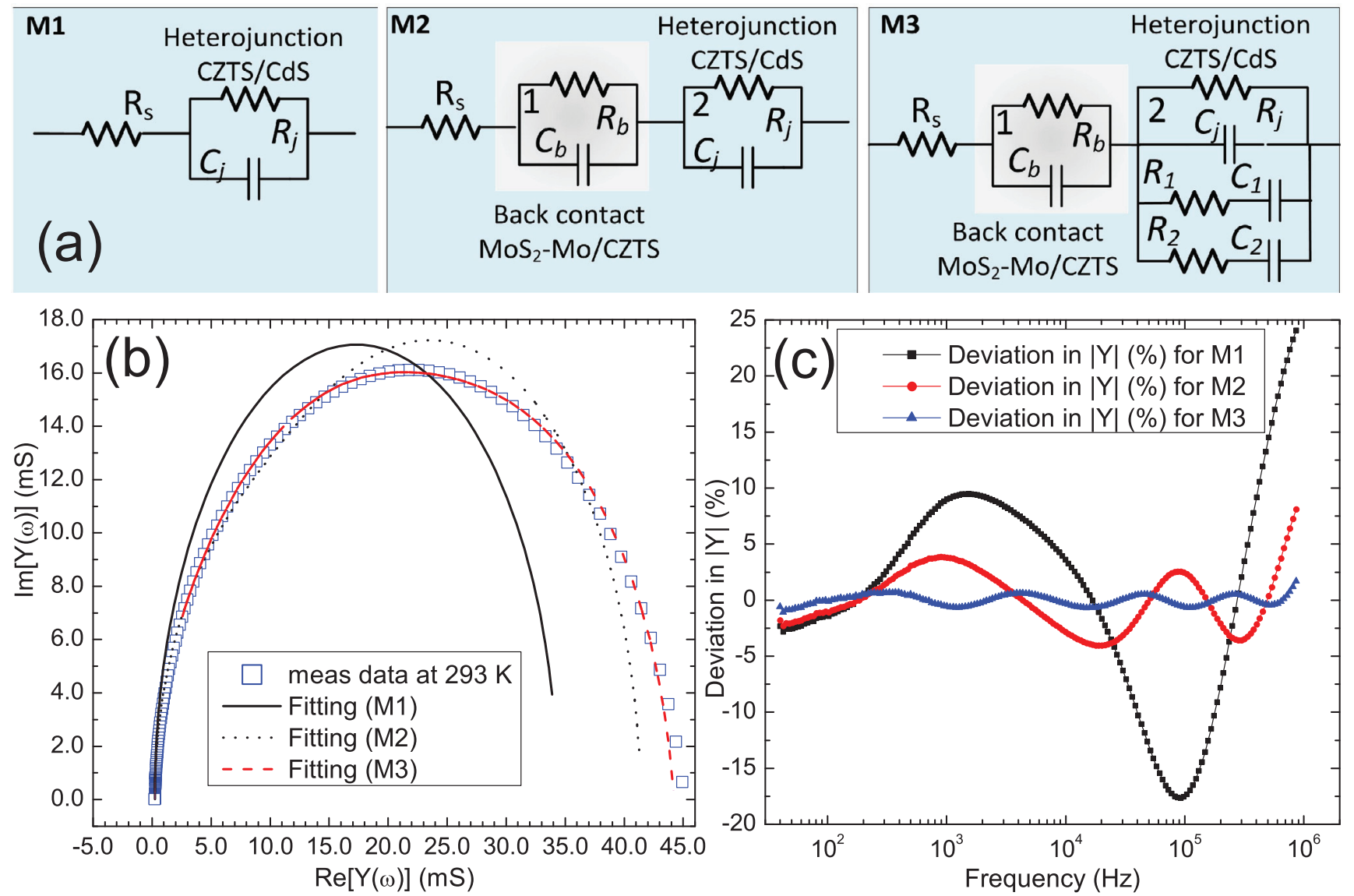

FIG. 2. (a) Tested AC response equivalent circuits of the studied solar cell. (b) Complex admittance plane of the studied solar cell measured at a temperature of $293 \mathrm{~K}$, in dark conditions with no applied bias voltage and the fittings results for the AC response equivalent circuits. (c) Fitting deviations of $|Y|$ vs. frequency for the three tested circuits. 

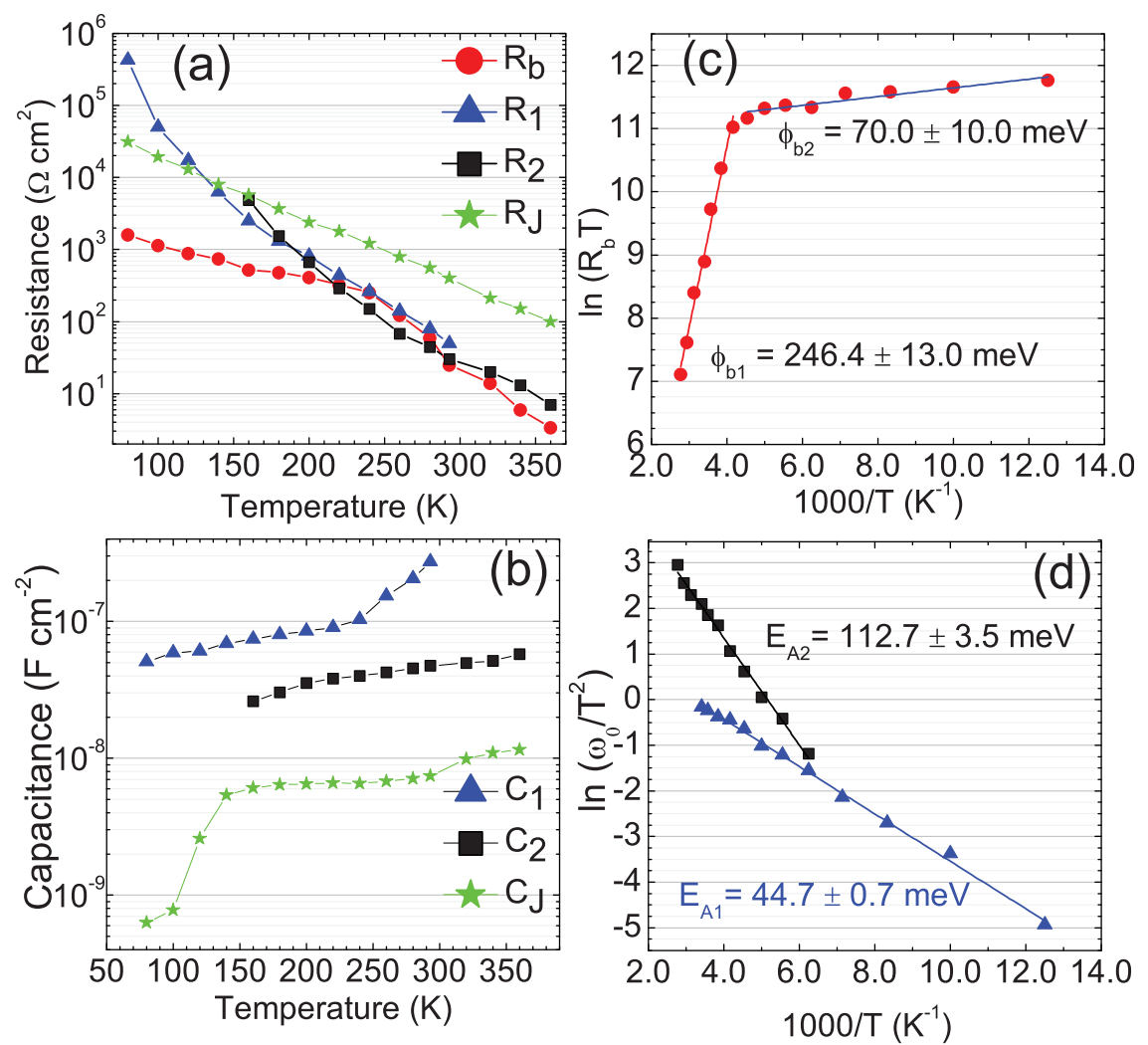

FIG. 3. (a) Extracted back-contact and trap related resistances for the temperature range $80 \mathrm{~K}-360 \mathrm{~K}$. (b) Extracted heterojunction and trap related capacitances for the temperature range $80 \mathrm{~K}-360 \mathrm{~K}$. (c) $\ln \left(R_{b} T\right)$ vs. $1 / T$ plot for the barrier height extraction. (d) Arrhenius plot for the estimation of the defect activation energies, $E_{A 1}$ and $E_{A 2}$. common on low mobility semiconductors. ${ }^{5}$ In fact, this feature was confirmed by Hall measurements in samples grown by a similar process. ${ }^{15}$ The temperature variation of the capacitances $C_{j}, C_{1}$, and $C_{2}$ is presented in Fig. 3(b). These variables show a decrease with decreasing temperature. The heterojunction capacitance, $C_{j}$, is characterized by a step decrease at $\simeq 280 \mathrm{~K}$, a plateau at $6.5 \pm 0.2 \mathrm{nF} \mathrm{cm}^{-2}$ and another step decrease at $\simeq 140 \mathrm{~K}$. The low temperature step may be explained by carrier freezing out and $C_{j}$ should tend to the heterojunction geometrical capacitance, which should be close to $0.5 \mathrm{nF} \mathrm{cm}^{-2}$. The other variables do not seem to have any distinguishable temperature dependence within the experimental apparatus resolution or it could be hidden below the measurements errors, $R_{s}=1.2 \pm 0.5 \Omega \cdot \mathrm{cm}^{2}$ and $C_{b}=98 \pm 25 \mathrm{nF}$ $\mathrm{cm}^{-2}$. According to Gunawan et al., ${ }^{4}$ the presence of a potential barrier can be defined by $R_{b}=\frac{k_{B}}{q A^{*} T} \times \exp \left(\frac{\phi_{b}}{k_{B} T}\right)$, where $A^{*}$ is the effective Richardson constant and $\phi_{b}$ is the barrier height at the interface. Fig. 3(c) presents the curve $\ln \left(R_{b} T\right)$ vs. $1 / T$, from which high temperature $\phi_{b 1}$ was extracted with a value of $246.4 \pm 13.0 \mathrm{meV}$. It is interesting to note that for a lower temperature the same curve also allow the estimation of a barrier with a different height, $\phi_{b 2}$, of $70.0 \pm 10.0 \mathrm{meV}$. The nature of this result is unclear but it could be related to the fact that the back contact device is formed by two interfaces, $\mathrm{MoS}_{2} / \mathrm{CZTS}$ and $\mathrm{Mo} / \mathrm{MoS}_{2}$. Attempts to model separately these two interfaces were not successful. Fig. 3(d) shows an Arrhnenius plot of the characteristic frequency, $\omega_{0}$, defined as $\omega_{0}=\frac{1}{R_{i} C_{i}}$, where $i=1,2$, for each trap level. The thermal emission depth of the defect, $E_{A}$, can be extracted following the expression $\omega_{0}=\xi_{0} T^{2} \times \exp \left(-\frac{E_{A}}{k_{B} T}\right)$, where $\xi_{0}$ is the thermal emission prefactor. ${ }^{16}$ The defect activation energies obtained were $(44.7 \pm 0.7) \mathrm{meV}$ and $(112.7 \pm 3.5) \mathrm{meV}$. The shallower defect, $E_{A 1}$, has a transition energy higher than the one obtained by Chen et al. of $\simeq 20 \mathrm{meV}$ for the $V_{C u}$ defect.
Photoluminescence studies of samples grown by a similar process pointed to defect activation energies values close to the ones of $E_{A 1} \cdot{ }^{15}$ On other hand, the deeper defect, $E_{A 2}$, seems to be close to the $C u_{Z n}$ defect activation energy of $120 \mathrm{meV}^{17}$

In summary, we propose an $\mathrm{AC}$ response equivalent circuit that consists of a series connection of three sections. The first loop comprises all series resistances of the device. The second describes the back contact behavior in the AC regime. The temperature study of the back contact shunt resistance shows a near room temperature barrier height of $246 \mathrm{meV}$. The third section describes the behavior of CZTS/ $\mathrm{CdS}$ heterojunction containing two trap levels. The analysis of the latter allowed the determination of a defect's activation energy of $45 \mathrm{meV}$ and $113 \mathrm{meV}$.

This work was supported by the PTDC/CTM-MET/ $113486 / 2009$ and PEst-C/CTM/LA0025/2011 projects funded by Fundação para a Ciência e Tecnologia (FCT). We would also like to acknowledge the financial support of FCT and the Brazilian agency CAPES to this project through the Grant Nos. 411.00 and 3779-08-4, respectively. The authors also thank A. Weber, C. Stephan, B.-A. Schubert, and R. Klenk from the Helmholtz-Zentrum Berlin for their help in the solar cells preparation.

${ }^{1}$ B. Shin, O. Gunawan, Y. Zhu, N. A. Bojarczuk, S. J. Chey, and S. Guha, "Thin film solar cell with $8.4 \%$ power conversion efficiency using an earth-abundant $\mathrm{Cu}_{2} \mathrm{ZnSnS}_{4}$ absorber," Prog. Photovoltaics (in press).

${ }^{2}$ D. A. R. Barkhouse, O. Gunawan, T. Gokmen, T. K. Todorov and D. B. Mitzi, Prog. Photovoltaics 20, 6 (2011).

${ }^{3}$ D. B. Mitzi, O. Gunawan, T. K. Todorov, K. Wang, and S. Guha, Sol. Energy Mater. Sol. Cells 95, 1421 (2011).

${ }^{4}$ O. Gunawan, T. K. Todorov, and D. B. Mitzi, Appl. Phys. Lett. 97, 233506 (2010).

${ }^{5}$ Y. Y. Proskuryakov, K. Durose, B. M. Taele, and S. Oelting, J. Appl. Phys. 102, 024504 (2007). 
${ }^{6}$ T. Walter, R. Herberholz, C. Müller, and H. W. Schock, J. Appl. Phys. 80, 4411 (1996).

${ }^{7}$ P. A. Fernandes, P. M. P. Salomé, A. F. da Cunha, and Björn-Arvid Schubert, Thin Solid Films 519, 7382 (2010).

${ }^{8}$ H. Katagiri, K. Jimbo, M. Tahara, H. Araki, and K. Oishi, Mater. Res. Soc. Symp. Proc. 1165, 1165-M04-01 (2009).

${ }^{9}$ P. A. Fernandes, P. M. P. Salomé, and A. F. da Cunha, Thin Solid Films 517, 2519 (2009).

${ }^{10}$ P. A. Fernandes, P. M. P. Salomé, and A. F. da Cunha, J. Alloys Compd. 509, 7600 (2011)

${ }^{11}$ B. Yeum, Electrochemical Impedance Spectroscopy Data Analysis Software, EChem Software, Ann Arbor, Michigan, USA, 2002.
${ }^{12}$ R. Sheer and H.-W. Schock, Chalcogenide Photovoltaics (Wiley-VCH Verlag \& Co. KGaA, Weinheim Germany, 2011).

${ }^{13}$ E. Barsoukov and J. R. Macdonald, Impedance Spectroscopy, Theory, Experiment, and Applications (John Wiley and Sons, Inc., Hoboken, New Jersey, 2005).

${ }^{14} \mathrm{M}$. Burgelman and P. Nollet, Solid State Ionics 176, 2171 (2005).

${ }^{15}$ J. P. Leitão, N. M. Santos, P. A. Fernandes, P. M. P. Salomé, A. F. da Cunha, J. C. González, G. M. Ribeiro, and F. M. Matinaga, Phys. Rev. B 84, 024120 (2011).

${ }^{16}$ T. Eisenbarth, T. Unold, R. Caballero, C. A. Kaufmann, and H.-W. Schock, J. Appl. Phys. 107, 034509 (2010).

${ }^{17}$ S. Chen, X. G. Gong, A. Walsh, and S.-H. Wei, Appl. Phys. Lett. 96, 021902 (2010). 